\title{
La palma de Moriche (Mauritia flexuosa L.f;) un ecosistema estratégico
} The Moriche palm(Mauritia flexuosa L. f) represents astrategic ecosystem

\author{
Juan M. Trujillo - Gonzalez ${ }^{1 *}$, Marco A. Torres Mora ${ }^{2 *}$ \\ Elvinia Santana - Castañeda ${ }^{3 *}$ \\ ${ }^{1}$ Ingeniero Agrónomo, Estudiante de Maestría en Ciencias Ambientales, UJTL \\ ${ }^{2}$ Biólogo, PhD. Profesor auxiliar Universidad de los Llanos \\ ${ }^{3}$ Bióloga, MsC. Profesora asistente Universidad de los Llanos \\ ${ }^{*}$ Grupo Biorinoquia \\ Email: juanm.trujillog@utadeo.ed.co
}

Recibido: Junio 10 de 2010. Aceptado: marzo 2 de 2011

\section{RESUMEN}

Con esta revisión se busca recoger los aspectos generales que se han investigado, especialmente en lo concerniente a la biología, la ecología y los usos, en los países donde se encuentra distribuida la especie.

La palma de moriche (Mauritia flexuosa L. f) presenta una amplia distribución desde la cuenca amazónica hasta la isla de Trinidad. La especie presenta una gran adaptación a suelos con baja fertilidad, además es parte integral del sistema natural regional porque se ha convertido en un elemento fundamental, para la conservación de las fuentes hídricas y la biodiversidad. En el caso de algunas economías locales es usada como un recurso que genera alimento, fibras y madera. Su importancia económica varía entre las diferentes regiones geográficas, en Colombia es escasamente comercializada, mientras que en zonas como lquitos (Perú), comienzan a seleccionar ecotipos para el cultivo comercial de la especie, ya que existe una red económica basada en sus productos.

Palabras clave: Mauritia flexuosa L.f, Aguaje, Amazonia, Orinoquia, relaciones ecológicas, usos tradicionales.

\section{ABSTRACT}

This review was mainly aimed at bringing togethergeneral aspects concerningthis speciewhichhave been investigated to date, especially regardingits biology, ecology and use in the countries where the specie is located. 
The Moriche palm(Mauritia flexuosa L.f) has a wide distribution, ranging from the Amazon Rever basin to the island of Trinidad. The specie has excellent adaptation to low-fertilitysoil; it is also an integral part of regional natural systems because it has become a fundamental elementin preserving hydric resources and biodiversity. It is being used as a resource generating food, fibres and woodin some local economies. Its economic importance variesamongst different geographical regions; it is barely commercialisedin Colombia while in zones like Iquitos (Peru), ecotypes have begunto be selected for the specie's commercial cultivation and exploitation as an economic network based on its productsalready exists.

Key words: Mauritiaflexuosa L.f, Aguaje, Amazonia, Orinoquia, ecological relationship, traditional use.

\section{Distribución natural}

La Mauritia flexuosa es una especie de la familia Arecaceae de acuerdo al sistema de clasificación propuesto por Uhl y Dransfield (1987), y Dransfield y Beentje (1996). El género Mauritia está restringido a la zona tropical del continente suramericano y aparentemente su centro de especiación fue la cuenca amazónica (Ponce 2000). La especie Mauritia flexuosa se expandió centripetamente a partir de la cuenca amazónica, colonizando las regiones bajas y mal drenadas de Suramérica (Muller 1970, González 1987). Actualmente la especie presenta una distribución a lo largo del norte de Sudamérica, oriente de los Andes, dónde forma a menudo grandes asociaciones en tierras ácidas y anegadas (Borgotoft y Baslev 1990), correspondientes a Brasil, Colombia, Bolivia, Ven- ezuela, Surinan, Guyana Francesa e Inglesa y Trinidad(Garzón y Leyva 1993; Domínguez 1995; Henderson et al. 1995; Cabrera y Wallace 2007).

Se afirma que el Moriche es de las pocas palmeras que puede crecer con el sistema radicular sumergido en los pantanos por poseer raíces aéreas llamadas neumatóforos (Del Castillos et al., 2006), de ahí, que el mayor centro de diversidad para la especie y el género se encuentre en el límite occidental húmedo de la hoya amazónica (Lleras et al,. 1993) (figura 1), y alcanza un rango de altitud que varía entre 5 a 1200 m.s.n.m (Cárdenas et al. 2007, Garzón y Leyva 1993, Ponce 2000) y con una precipitación anual media en su área de distribución que varía entre 1141-6315 mm. y con temperaturas anuales entre $22,8-27,1^{\circ} \mathrm{C}$ (Borgtoft y Balslev 1990).

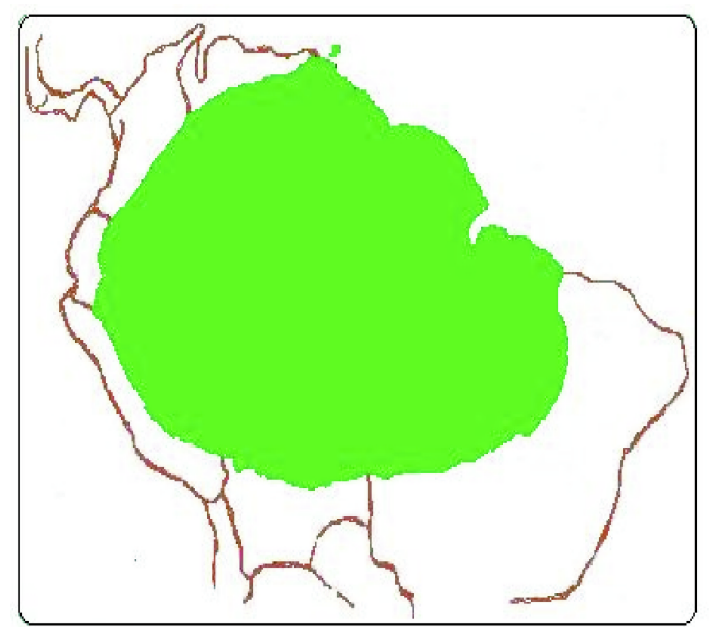

Tomado de: Henderson et al. 1995

Figura 1. Distribución de la Mauritia flexuosa L. $f$ 
En Colombia la especie se encuentra distribuida en los Llanos Orientales, en el piedemonte andino y en formaciones de sabanas y selva húmeda del Vaupés, Amazonas, Guainía, Guaviare, Caquetá, Vichada y Putumayo (Bohórquez 1976; Garzón y Leyva 1993).

La Mauritia flexuosa recibe su nombre en homenaje a Johan Mauritz van Nassau-Siegen, rey de los países bajos, entre 1567-1623 (Bohórquez 1976) y se conoce con los siguientes nombres comunes: en Colombia moriche en los llanos orientales, canangucha, cananguche o canangucho en los departamentos de Caquetá y Putumayo (Galeano y Bernal 2010); burití y miriti en Brasil, moriche en Venezuela, aguaje en Perú, morete en Ecuador y palma real en Bolivia.

\section{Aspectos Morfológicos}

La Mauritia flexuosa es una planta dioica, arborescente monocaule que puede alcanzar una altura de 40 metros, como se reporta para la región amazónica brasileña (Storti 1993), un diámetro de 30 a $60 \mathrm{~cm}$, y finaliza en una corona de 15 a 20 hojas costapalmadas dispuestas en espiral, de 2,5 metros de largo y $4,5 \mathrm{~m}$. de ancho, con sus hojas senescentes persistentes; presentan una vaina que cubre parcialmente el tallo (Galeano 1991, Henderson et al (1995).

Las inflorescencias masculinas y femeninas son de tipo interfoliares y semejantes. Las masculinas presentan un raquis leñoso y cilíndrico de longitud promedio de 3,23 metros, que puede ir de 2,37 a $4,79 \mathrm{~m}$ (Storti 1993); con 36 ramas dísticas, raquis secundarios leñosos comprimidos de 0,8 a $1 \mathrm{~m}$. de largo y cubiertos de brácteas cónicas (Henderson et al. (1995), y se producen entre 4 a 7 raquis por año (Ponce 2000, Ponce 2002). Las inflorescencias femeninas presentan de 2-8 por raquis por palma (Cárdenas et al. 2007) con un promedio de longitud de 2,44 metros de longitud promedio, con una variación entre 1,68 y 3 metros, las raquillas puede tener entre 0,20 y 1,37 metros de longitud y se estiman por raquilla 3,612 flores entre $1,7 \mathrm{~cm}$ de ancho por $1,2 \mathrm{~cm}$. de largo, que producen en promedio 479 frutos (Storti 1993). Las flores femeninas se caracterizan por presentan cáliz 64 campanulado, corola tripartida, gineceo súpero, ovario triloculado, óvulos ortóptropos y estigma sesil.

Los frutos son una drupa, elíptica u oblonga de 6 a $7 \mathrm{~cm}$. de longitud de color café-rojizo cuando alcanzan la madurez, con un peso que varía entre los 15 a 120 g., con un promedio de 50g. (Restrepo y Duran 1988), constituidos por un exocarpio con escamas imbricadas y fuertemente soldadas, un mesocarpio carnoso de color anaranjado y espesor variable, y endocarpio pergaminado. La semilla presenta una forma subglobosa con un endospermo homogéneo.

El moriche presenta un tapete de raíces que crece 20 centímetros por debajo del suelo y alcanza una distancia horizontal de 40 metros cubriendo un área de más de 5000 metros cuadrados (Del Castillo et al. (2006), con raíces secundarias aéreas llamadas neumatóforos importantes en el intercambio de gases sobre la superficie de la tierra (Borgtoft y Balslev 1990), lo que concede al moriche superioridad competitiva por espacio y aire y posibilita el establecimiento de rodales puros en zonas desfavorables a otras especies (Granville 1974, Balick 1979).

\section{Relaciones ecológicas}

El moriche es una palma gregaria que forma asociaciones vegetales homogéneas a lo largo de ríos y caños denominadas "morichales" donde llega a ser la especie dominante del estrato arbóreo. EI resto de la formación vegetal está compuesta por arbustos, estrato bajo de ciperáceas, helechos, bromeliáceas y briofitos entre otros (Galeano 1991). Kahn (1988), Borgotoft y Balslev (1990), indican que la Mauritia flexuosa se encuentra a menudo en posiciones monoespecíficas extensas o junto con otras palmas como la Oenocarpus batua y Euterpe precatoria.

San José et al. (2009), encontraron que en las zonas ecotonales de los morichales se presenta una gran variedad de especies vegetales donde se destacan las familaias Poaceae (Acroceras zizanoides, Axonopus compressus, Isachne polygonoides, Panicum cayenensis y P. parviflorum), Cyperaceae (Eleocharis interstinta, E. plicarhachis, 
Rhynchospora papilosa), Melastomataceae (Rhynchanthera serrulata), Labiatae (Hyptis conferta), Xyridaceae (Xyris savanensis), y Eriocaulaceae (Syngonanthus caulencens).

La Mauritia flexuosa L. f., es uno de los recursos alimenticios utilizados por guacamayos y loros, tapires, pecaries, peces, tortugas y monos (Goulding 1989, Bodmer 1990, Bonadie \& Bacon 2000, Brightsmith y Bravo 2006), que a su vez se convierten en dispersores de las semillas, en las regiones donde ésta se distribuye. (Urrego, 1987; Da Silva, 2009)

VanAndel (1990), en la "Caracterización y clasificación de bosques inundables en una llanura aluvial en el medio Caquetá, Amazonas (Colombia)", encontró que en zonas mal drenadas con inundación permanente por lluvia y agua de pequeñas quebradas de origen amazónico, domina la M. flexuosa L. f., que representa hasta $75 \%$ del área basal total y con un dosel superior abierto de $20 \mathrm{~m}$. de altura en promedio. En la sabana la especie también domina el dosel superior y alcanza un $24 \%$ de área basal total.

En la altillanura disceptada colombiana el moriche se distribuye a lo largo de las zonas de drenaje, mientras que en la altillanura plana se asocia a arroyos pequeños donde puede hacer parte de los bosques riparios. La comunidad de morichal juega un papel importante como protectora de cauces de agua permanente, particularmente en regiones donde existe una estacionalidad climática marcada (Ponce, 2002).

\section{Fenología}

La fenología es el estudio del comportamiento de las plantas en relación a su entorno, abarca patrones estacionales de floración, fructificación y producción de follaje (Rojas y Da Cruz 2004). Sarmiento y Monasterio (1983), consideran que el comportamiento fenológico de ciertas especies en un ecosistema dado, debe considerarse como un componente parcial de su estrategia evolutiva global, lo que significa que los mecanismos de adaptación de una población son una respuesta relativa de los individuos a cada una de las fases de su desarrollo y a los impulsos y fuerzas del ambiente.
En la región de Manaos (Brasil), la floración en palmas masculinas se presenta en el periodo de febrero a agosto, con un pico en el mes de abril, mientras que en las femeninas ocurre principalmente entre los meses de abril a junio (Storti 1993). Según Cabrera y Wallace, (2007), en el departamento de la Paz (Bolivia), la floración tanto de hembras y machos presenta sincronía, con picos máximos entre octubre y noviembre. Para la región amazónica colombiana el inicio de la floración se sitúa entre julio y agosto, es simultáneo en morichales de áreas y condiciones fisiográficas diferentes como sabanas, rebalses e islas (Urrego 1987), y coincide con el final de la principal estación lluviosa, inundación máxima, mínimas horas de sol por día, mínimo promedio de temperatura anual y mínima diferencia en las temperaturas diarias (Borgtoft y Balslev 1990).

En el delta del Orinoco (Venezuela) existen dos periodos de floración para $M$. flexuosa L. f., uno con mayoría de flores estaminadas en el mes de mayo, cuando se presenta la principal temporada de lluvias, y otro con flores pistiladas en diciembre, asociado a menores precipitaciones (Heinen y Ruddle 1974).

La producción de frutos puede variar entre 9 y 12 meses desde la floración hasta el desprendimiento de los frutos, fenofase que se presenta cada dos años por individuo, pero que a nivel poblacional es anual. Es decir, que si bien las poblaciones son sincrónicas en los procesos de fructificación, a nivel de individuo existe asincronía de un año a otro (Storti 1993, Ponce 2002).

En la región del Orinoco el desarrollo de los frutos maduros necesita de cuatro a cinco meses después de florecer y se encuentran durante todo el año, con una marcada cresta entre julio a octubre y una menor entre febrero a abril (Heinen y Ruddle 1974, Ponce, 2002), mientras que para la Amazonía peruana se reporta carencia de frutos entre agosto y noviembre (Ruiz, 1993).

Triana (1998), en "experiencias con palma de Canangucha (Mauritia flexuosa L.f.) del piedemonte caqueteño", encuentra una estrecha relación entre la dinámica hídrica y los ciclos fenológicos de la especie. La mayor floración se presenta entre los 
meses de julio a agosto (meses secos y con vientos), mientras que la fructificación y maduración de frutos sucede aproximadamente siete meses después, cuando las condiciones de encharcamiento son propicias para la germinación de los frutos caídos; información complementada por las poblaciones nativas de la región que reportan que la fructificación de la canangucha están desde el mes de octubre hasta julio o agosto. (Cárdenas et al., 2007)

\section{Usos tradicionales}

El moriche se constituye en el recurso económico y social de mayor importancia para muchas poblaciones amazónicas (Vásquez et al,. 2008). En la Amazonía baja peruana su fruto se constituye en el más importante del mercado urbano y proporciona ingresos significativos a la población, principalmente mujeres (Del Castillo et al., 2006). En Venezuela las comunidades indígenas usan la masa seca como "pan" (harina), en Brasil se convierte en la mayor fuente alimenticia para poblaciones ribereñas del Estado do Pará y en Ecuador juega un papel importante en la alimentación de poblaciones nativas.

La pulpa de $M$. flexuosa L. f. es una de las más nutritivas del trópico, de donde se puede extraer vitaminas, lípidos, proteínas, minerales, entre otros (Vásquez, et al. 2008). En cuanto a su contenido de aceite alcanza del $12-24 \%$ y 2,3-3,7 \% de proteína, un alto contenido de fósforo $(27 \mathrm{mg} / 100 \mathrm{~g}$. de pulpa) (Anónimo 1983) y la mayor tasa de provitamina $A(4,6 \mathrm{mg} / 100 \mathrm{~g}$. de pulpa) encontrada en la naturaleza (Storti, 1993). En frutos recolectados en la altillanura colombiana se encontraron puntos porcentuales de proteína y grasas de 5,4 y 4,28 respectivamente (Torres, 2003).

La Mauritia flexuosa L.f, al igual que otras especies de palmas son fuente de aceites y grasas que se extraen del mesocarpo de los frutos maduros mediantes procesos sencillos realizados por campesinos e indígenas. El aceite de moriche tiene un alto contenido en ácido oleico equivalente al de otras especies oleaginosas y palmas nativas, (Tabla 1 ).

Tabla 1. Porcentaje de ácidos grasos* en aceites de tres especies de palmas

\begin{tabular}{lccc}
\hline $\begin{array}{c}\text { Ácido } \\
\text { graso }\end{array}$ & Moriche & Seje & $\begin{array}{c}\text { Palma de } \\
\text { aceite }\end{array}$ \\
\hline Láurico & ------ & ----- & 0,35 \\
Mirístico & ----- & ----- & 1,09 \\
Palmítico & 15,45 & 10,49 & 40,51 \\
\hline Esteárico & 1,41 & 3,53 & 3,9 \\
\hline Oleico & 79,33 & 80,08 & 41,47 \\
\hline Linoleico & 1,32 & 3,29 & 11,04 \\
\hline Linolénico & 1,06 & 0,83 & 0,26 \\
\hline Cronomatografía, Método U.S.P Tomado de: Torres, 2003
\end{tabular}

En lquitos (Perú) de acuerdo al color y al espesor de la pulpa, los pobladores reconocen las siguientes variedades de M. flexuosa (Ruiz 1993; Delgado et al, 2007):

\footnotetext{
- "Aguaje Shambo": pulpa rojiza, preferido por su sabor agradable

- "Aguaje ponguete": mesocarpio amarillo

- "Aguaje carnoso": pulpa gruesa (espesa)
}

Del aguaje maduro se obtine la "pasta" (pulpa sin semilla) de donde se elabora la "aguajina" (bebida denominada "leche de la amazonia"), el "chupete" y el "helado de aguaje" entre otros; de su semilla se obtiene "marfil vegetal" que es utilizado para la elaboración de utensilios domésticos, botones y artesanías (Ruiz 1993, Rojas et al., 2001).

Las hojas son usadas para techos de casas y producción de fibras, cordeles, sogas, hamacas, 
esteras, canastos, redes de pesca y atuendos ceremoniales (Rondón 2005; Schmidt et al., 2007), Figueredo et al. (2007) recomienda coletar 2 hojas por planta cada ochos mes. En cuanto a posibles usos industriales de las hojas de moriche, De los Heros \& Zárate 1981, en su investigación "Posibilidades papeleras de pulpa al sulfato de pecíolos de Aguaje (Mauritia flexuosa)", encuentran que pueden ser utilizadas como materia prima en la industria del papel y del cartón (De los Heros y Zárate 1981).

Los tallos al igual que las otras partes de la planta tienen una variedad de usos que van desde la producción de alimento (almidón y vino), materiales para construcción (tablones y postes) y fabricación de canoas entre otros. Las tribus indígenas y colonos utilizan los troncos de las plantas muertas para la cría de las larvas del Rhynchophorus palmarum, que se constituye en alimento de alta calidad por su contenido proteico y energético, debido a la acumulación de grasa que presenta. De la yema apical se obtiene el palmito, de menor calidad que el de otras especies de palmas, consumido cocinado en ensaladas o encurtidos.

\section{Cultivo}

En las regiones donde se distribuye la $M$. flexuosa L.f, por lo general se hace una explotación de tipo extractivo; la cosecha se realiza cortando la palma cuando los frutos se tornan de color marrón intenso y se desprenden fácilmente, este manejo afecta la densidad poblacional y pone en peligro la supervivencia de la especie.

Urrego (1987) en la región de Araracuara (Caquetá) se encuentra que en forma silvestre y espaciada irregularmente se alcanzan producciones de frutos estimadas en 9,07 Ton/ha, lo que la amerita a esta palma como una especie nativa promisoria para su explotación comercial (Urrego, 1987). En evaluaciones realizadas en morichales del pie de monte caqueteño, Triana (1998) halló un promedio de producción por cosecha de 3,5 racimos, con un peso individual de 60 libras (solo frutos) por planta. En el cerrado brasileño una planta puede producir 4000 frutos, estimando una producción de 6.1 ton/ ha/año (Da silva, 2009).
En plantaciones con 100 palmas de moriche por hectárea y espaciamientos de $10 \times 10 \mathrm{~m}$. realizadas en Perú, se obtuvo un rendimiento de 19 Tn/ha, que traducidas a kilocalorias/ha representaría 10,868 (Bohórquez 1976). Lleras et al (1988), estimaron que con una producción de 200 kilogramos de frutos por palma se lograrían 24 kilogramos de aceite (sistema extractivo). La produccion de frutos comienza a partir de los 7 u 8 años y una altura entre 6 y 7 metros (Delgado y Couturier, 2003).

Sobre la agronomía de esta especie se ha realizado estudios de evaluación y selección de germoplasma con el fin de lograr un mejoramiento genético que permita su implementación como cultivo comercial (Anónimo 2006, González et al. (2006). El hecho de ser una planta generalmente dioica ofrece dificultades de plantación, tanto por la identificación de los sexos, como por la identificación de la proporción adecuada de éstas. En Perú el desarrollo del cultivo de la especie se viene adelantando con un ecotipo denominado "enano", el cual no necesita de suelos con altos niveles de humedad (Vásquez, et al., 2008).

Con la implementación del aguaje como cultivo comercial han surgido diversas necesidades de investigación que permitan conocer los factores que afectan la producción, como en el caso las plagas donde la información sobre los daños que ocasionan al cultivo es todavía muy escasa (Villachica, 1996). Vásquez et al (2008) en la región de lquitos encontraron y clasificaron las especies de insectos presentes en las plantaciones comerciales y jardines de aguaje enano. La investigación clasificó las especies de acuerdo si el daño ocasionaba pérdidas en la producción o si era insignificante para la cosecha de este ecotipo. Para el primer caso, donde afectan los frutos, las flores y los raquis se encontraron las especies Eupalamides cyparissias Fab, Antiteuches kerzhneri Rider, Stenoma sp., Clinodiplosis sp., Cecidomyidae sp, Cucurlionidae sp 1., Cucurlionidae sp 2. y Tyrannion sp. Las especies que actúan a nivel de hojas, estípites y raíces, cuyo daño no se consideró significativo, son Brassolis sophorae L., Opsiphanes cassina Felder, Leptoglossus hesperus Brailosky \& Couturier, Ceratiphis brasiliensis Hempel, Rhynchophorus palmarum L, Rhinostomus barbirostris Fab, Metamasius hemipterus L., strategus surinamensis Burmeister, Atta cephalontes L. y Trigonasp. 
De igual manera se viene trabajando con la $M$. flexuosa L. f. en sistemas agroforestales, con el fin de mantener una alta diversidad de cultivos tropicales que favorezcan el potencial económico y ecológico del recurso; algunas de estas combinaciones son aguaje con yuca y maíz, aguaje con caña de azúcar y aguaje con maní, sandía y papaya.

\section{Conclusiones y perspectivas de investigación}

Mauritia flexuosa L. f, se distribuye a lo largo de zonas de drenaje y juega un papel importante en la protección del recurso hídrico en regiones con estacionalidad marcada, como es el caso de la altillanura colombiana, además ocupa un lugar importante en la alimentación de la fauna que se asocia a estas comunidades.

La palma de moriche, es una fuente de recursos reconocida en la amazonia peruana donde se constituye en una de las especies vegetales más populares por la serie de productos que de ella se obtienen, como la pulpa para bebidas, helados y sorbetes; el marfil vegetal de su semilla que es utilizado en la elaboración de artesanías; las fibras provenientes de sus hojas para tejidos y sus hojas completas para la cubierta de las construcciones. Todos estos usos diversos, la convierten en uno de los recursos que sustentan economías locales y valores culturales. En la Orinoquia colombiana, el

\section{REFERENCIAS}

Anónimo.1983. FAO/CATIE. Informe de la reunión de consulta sobre palmeras poco utilizadas de América tropical. Turrialba. Costa Rica.

Anónimo. 2006. PERÚ. INCAGRO. Domesticación y servicios ambientales del aguaje (Mauritia flexuosa L. f.) en la amazonía peruana. Iquitos.

Castaño N, Cárdenas D, Octavo E. Editores. 2007 Ecología, aprovechamiento y manejo sostenible de nueve especies de plantas del departamento del Amazonas, generadoras de productos maderables y no maderables. Instituto Amazónico de Investigaciones Científicas - Sinchi-Corporación para el Desarrollo Sostenible del Sur de la Amazonia CORPOAMAZONIA. Bogotá, Colombia. moriche está restringido a usos locales, como la utilización de sus hojas para techos de casas en zonas rurales y lugares turísticos de centros urbanos.

Para obtener la materia prima, frutos y hojas especialmente, se utiliza por parte de las comunidades técnicas de recolección inadecuada. En el caso de las hojas se extrae una alta cantidad que afecta las funciones fisiológicas normales de la planta; mientras que para la obtención de frutos se hace una poda radical de la palma femenina, dejando las comunidades con una mayoría de plantas masculinas, lo que afecta el repoblamiento natural.

Dentro de un modelo futuro de desarrollo integrado será necesario ofrecer alternativas combinadas de conservación y uso que permitan garantizar la sobrevivencia de los morichales; más aún, que la palma de moriche al ser hospedera de diversas especies de insectos, que para ella no representa mayor riesgo, si son plagas importantes en los cultivos de palma de aceite.

Si se tiene en cuenta los diferentes usos que se da a la especie, no se exagera al presentarla como una de las palmas con gran potencial para ser utilizada tanto en la agroindustria, la farmacéutica, la jardinería y en la recuperación de suelos y la protección del recurso hídrico, entre otros.

Balick M. Amazonian oil palms of promise: A survey. Economic Botany 1979; 33(1): 11-28.

Bodmer R. Responses of angulates to seasonal inundations in the amazon floodplain. T. Trop. Ecol 1990; 6: 191-201.

Bohórquez JA. 1976. Monografía sobre Mauritia flexuosa. Pp: 233 -245 en C Villegas (ed).Simposio Internacional sobre plantas de interés económico de la Flora amazónica-IICA-TROPICOS. Turrialba, Costa Rica.

Bonadie W, Bacon P. Year-around utilization of fragmented palm swamp forest by Red- Bellied Macaws (Ara manilata) and Orange-winged parrot 
(amazona amazonica) in the Neriva (Trinidad) Biol. Conserv. 2000; 95: 1-5.

Borgotoft P, Balslev H. 1990. Ecuadoreans Palms for agroforestry, AAU Reports 23. Botanical Institute Aarhus University. Pontificia Universidad católica de Ecuador. Quito. 117 pp.

Brightsmith D, Bravo A. Ecology and Management of nesting blue-and-yellow macaws (Ara ararauna) in Mauritia palm swamps. Biodiversity and Conservation 2006; 15:4271-4287.

Cabrera H, Wallace R. Patrones fenologicos de ocho species de palmeras en el bosque amazónico de Bolivia. Rev. Bol. Ecol y Cons. Amb. 2007; 21: 1-18.

Da Silva P. 2009. Orthopsittaca manilata (Boddaer, 1783) (Aves: Psittacidae): Abundância e atividades alimentar em relação de Mauritia flexuosa L. f. (Arecaceae) numa vereda no Triângulo Minero. Tesis de Maestria. Universidad Federal de Uberlândia. Brasil.

De Los Heros M , Zárate J. Posibilidad papelera de pulpaal sulfato de pecíolos deaguaje (Mauritia flexuosa L. f.). Revista forestal del Perú 1981; 10 (1-2): 83-90.

Del Castillo T, Otalora E, Freitas L. 2006. Aguje: la maravillosa palmera de la amazonía. Instituto de Investigaciones de la amazonía Peruana. 54pp.

Delgado C, Couturier G. 2003. Relationship Between Mauritia flexuosa and Eupalamides cyparissias in the peruvianAmazon. Palms. Volume 47-2.

Delgado C, Couturie G, Mejia K. 2007. (Arecaeae: Calamoideae), an amazonia palm with cultivation porposes in peru. Fruits. Vol 62 (3).

Domínguez A. 1993. Ensayos de procesamiento de "Pijuayo" (Guilielma gasipaes B.K. - L.H. Bailei) y "Aguaje" (Mauritia flexuosa). Pie de imprenta - Iquitos: Instituto de investigaciones de la Amazonia Peruana.

Dransfield J, Beentje H. 1996. Lexicon palmarum. The Royal Botanic Garden Kew. Editions Champflour, 64 pp. France.

Figueiredo I, Schmidt I, Sampaio M, ScariotA. 2007. Documentos de trabajo. Manejo sustentável de capim dourado e buriti no jalapão, Tocantins. Brasilia.
Galeano G. 1991. Las palmas de la región de Araracuara. Amazonia colombiana. Tropenbos-Colombia. Bogotá.

Galeano G, Bernal R. 2010. Palmas de Colombia. Guía de campo. Editorial Universidad Nacional de Colombia. Instituto de Ciencias Naturales, Facultad de Ciencias, Universidad Nacional de Colombia, Bogotá. 688 p.

Garzón C, Leyva P. 1993. Perfiles sintéticos sobre especies amazónicas con potencial económico. Perfil N.-28: Mauritia flexuosa Linn. F. Universidad Nacional de Colombia - Instituto de Ciencias Naturales, programa de Botánica económica - Corporación Aracuara - Proyecto Tropenbos. Bogotá.

Gonzalez A, Jarama A, Chuquival G, Vargas R. Colección y evaluación de germoplasma de (Mauritia flexuosa L. f.) aguaje en la amazonía peruana. Folia amazónica 2006;15:1-2.

González BVC. 1987. Los morichales de los Llanos Orientales: Un enfoque ecológico. -CORPOVEN. Caracas. $56 \mathrm{pp}$.

Goulding M. 1989. Amazon: The Foofen forest. BBC Book. London. Uk.

Granville JJ. 1974. Apercur sur la structure des pneumatophoros de deux espeses des sols hydromorphes en Guayana, Cah. OROTOM. Ser Biol $N^{\circ} 23: 3-2$.

Heinen H, Ruddle K.. Ecology, ritual and economic organization in the distribution of palm starch among Warao of the Orinoco delta. J. Anthropol. Res. 1974; 30: 1 16-1 38.

Kanh F. 1988. Ecology of economicaly important Palms in peruvian amazonia. En: Advances in Economics Botany 6:42-49. New York.

Lleras E, Giacometti DC, Coradin L. 1983. Areas criticas de distribución de palmas en las Americas para colecta, evaluación y conservación. Pp. 67-101. en: Informe de la reunión de consulta sobre palmeras poco utilizadas de America Tropical. FAO, Turrialba. 
Muller J. Palinological evidents on early differentions of angiosperms. Biological Review 1970; 45: 417-450.

Ponce M. 2000. Algunos aspectos de la biología poblacional de Mauritia flexuosa L. f. (palma moriche) en los llanos sur orientales del Estado Guárico, Venezuela. Tesis doctoral. Facultad de Ciencias. Universidad Central de Venezuela. Caracas.

Ponce M. 2002. Patrón de caída de frutos en Mauritia flexuosa L. f. y fauna involucrada en los procesos de remoción de semillas. Acta botánica venezuelica 25(2) Caracas.

Restrepo J, DuránE. 1988. Proyecto de investigación: evaluación físico-química y nutricional de las diferentes especies de palmas amazónicas en la región de Araracuara. Universidad del Valle. Calí.

Rojas R, Da Cruz J. Comportamento fenológico da palmeira patauá (Oenocarpus bataua) na reserva florestal Adolpho Ducke, Manaus, Amazonas, Brasil. Acta Amazonica 2004; 34 (4): 553-558.

Rojas R, Salazar C, Llerena C, Rengifo C, Ojanama J, Muñoz V, Luque H, Solignac J, Torres D, Panduro F. Industrialización primaria del aguaje (Mauritia flexuosa L. f.) en lquitos Perú. Folia amazónica 2001; 12:1-2.

Rondón J. 2005. Especies vegetales de uso en la cestería por la etnia Piaroa del Estado Amazonas, Venezuela. Revista Chapingo serie ciencias forestales y del ambiente Vol. XI N.- 002. Pp. 131 - 138. Universidad Autónoma de Chapingo. Chapingo, México.

Ruiz J. 1993. Alimentos del bosque amazónico: una alternativa para la protección de los bosques tropicales. UNESCO, oficina regional de ciencia y tecnología. Montevideo.

San Jose J, Montes R, Mazorra M, Matute N. Heterogeneity of the inland water-land palm ecotones (morichals) in the Orinoco lowlands, South America. Plan Eco. 2009; 208 (2): 1-11.
Sarmiento G, Monasterio M. 1983. Life forms and phenology. F Bourliere (ed) Tropical savannas. Ecosystems of the word. Vol 13. Amsterdam.

Schmidt I, Figueredo I, Sampaio M. 2007. Capim dourado e buriti: práticas para garantir a sustentabilidade do artesanato. PEQUI - Pesquisa e Conservação do Cerrado. Brasilia.

Storti E. Biología floral de Mauritia flexuosa L. f. na regiao de Manaus, AM, Brasil. ActaAmazónica 1993; 23(4): 371-381.

Torres M. 2003. Producción de biodiesel de moriche (Mauritia flexuosa L. f) como alternativa energética para las regiones apartadas de la Orinoquia colombiana. Tesis doctoral. Facoltá di Ingegneria. Universitá di Roma "La Sapienza". Roma.

Triana M. 1998. Experiencias con palma canangucha (Mauritia flexuosa L. f) del proyecto pie de monte caqueteño. En: Memorias taller sobre palmas amazónicas. Corpoica, regional Amazonia. Florencia.

Uhl N, Dransfield J. 1987. Genera Palmarum. Aclassification of Palms based on the work of $\mathrm{H}$. E. Moore Jr. L. H. Bailey Hortorium and the International Palm Society. Allen Press, Lawrence, Kansas. 610 pp.

Urrego L. Estudio preliminar de la fenología de la canangucha (Mauritia flexuosa L. f.). Colombia amazónica, Vol. 1987; 2 (2): 1987.

Van Andel T. 1990. Caracterización y clasificación de bosques inundables en una llanura aluvial en el medio Caquetá, Amazonas, Colombia. Tesis de Maestría. University of Amsterdam.

Vásquez J, Delgado C, Couturier G, Mejia K, Freitas L, Del Castillo D. 2008. Pest insects of the palm tree Mauritia flexuosa L.f., dwarf form, in peruvian amazonia. Fruits. Vol. 63 (4).

Villachica M.1996. Frutales y hortalizas promisorias de la amazonia, TCA, Perú. 